\title{
SUNED Research

\section{El Aprendizaje Basado en Problemas como estrategia didáctica-evaluativa en la enseñanza universitaria de la historia}

\author{
Humberto Andrés Álvarez Sepúlveda ${ }^{1}$ \\ 1. Universidad Católica de la Santísima Concepción, Departamento de Didáctica de la Facultad de Educación, Chile; \\ halvarez@ucsc.cl
}

Recibido 20-V-2020 • Corregido 28-VII-2020 • Aceptado 30-VII-2020

DOI: https://doi.org/10.22458/urj.v12i2.2906

\begin{abstract}
Problem-Based Learning as a didacticevaluative strategy for teaching history at the university level". Introduction: One of the main challenges of history teaching at the university is to counteract "teacheroriented" education with active methods, such as Problem-Based Learning, which allow teachers in training to develop historical competencies and soft skills relevant to their future professional performance. Objective: Evaluate Problem-Based Learning in history professor training, in five courses. Methods: I tested the method between March and December 2019 in 144 Pedagogy students from a Chilean university. The instruments for data collection were the Likert satisfaction survey, the students' field notebooks, the attendance record, the rubric designed to evaluate the method and the researcher's diary. Results: Overall, the students were satisfied with the method. However, there were also some limitations, including the students' lack of familiarity with Problem-Based Learning, the lack of group cohesion and the heavy workload that it requires. Conclusions: The development of soft skills was consistent with reports from other fields, but apparently this is the first time the method was tried in the field of history didactic.
\end{abstract}

Keywords: Problem-based learning (PBL), initial teacher training, Teacher-centered instruction, historical laboratory, historical thinking.
RESUMEN. Introducción: Uno de los principales desafíos de la enseñanza de la historia en la universidad es contrarrestar la educación "centrada en el docente" con métodos activos, como el Aprendizaje Basado en Problemas, que permiten a los docentes en formación desarrollar competencias históricas y "habilidades blandas" relevantes para su futuro desempeño profesional. Objetivo: Evaluar el aprendizaje basado en problemas en la formación de profesores de historia, usando cinco cursos. Métodos: Probé el método entre marzo y diciembre de 2019 en 144 estudiantes de pedagogía en una universidad chilena. Los instrumentos de recolección de datos fueron una encuesta de satisfacción Likert, los cuadernos de campo de los estudiantes, el registro de asistencia, la rúbrica diseñada para evaluar el método y el diario del investigador. Resultados: En general, los estudiantes se mostraron satisfechos con el método. Sin embargo, también hubo algunas limitaciones, incluida la falta de familiaridad de los estudiantes con el Aprendizaje Basado en Problemas, la falta de cohesión grupal y la gran carga de trabajo que requiere. Conclusiones: El desarrollo de habilidades blandas fue coherente con informes de otros campos, pero aparentemente esta es la primera vez que se prueba el método en el campo de la didáctica de la historia.

Palabras clave: Aprendizaje basado en problemas (ABP), formación inicial docente, magistrocentrismo, laboratorio histórico, pensamiento histórico.

La formación del profesorado de historia requiere una mayor atención de parte de las universidades, ya que resulta fundamental que aumenten sus esfuerzos para preparar a docentes capaces de afrontar los nuevos cambios que suceden en la educación superior y en el mercado laboral del siglo XXI (González, Santisteban, \& Pagès, 2020; Sáez, Parra-Díaz, \& Herrera, 2020). Entre estos, como señalan Gómez, López, Miralles y Prats (2017), destaca la necesidad de reorientar la enseñanza universitaria de la historia hacia enfoques didácticos más constructivistas que ayuden a los profesores en formación a convertir a las nuevas generaciones de estudiantes en ciudadanos responsables, reflexivos y solidarios con sus pares.

A pesar de lo anterior, el magistrocentrismo sigue prevaleciendo en las aulas universitarias, puesto que el académico formador, de forma habitual, se muestra reticente al momento de implementar nuevas estrategias didácticas. Por tal motivo, diversos autores como Barton (2010), 
Barca (2011) y Prats (2017) sostienen que los estudiantes suelen concebir la historia como una asignatura aburrida, memorística y escasamente útil para la vida cotidiana.

A raíz de dicha situación, es indispensable innovar en la clase de historia a partir de la implementación de metodologías activas que posibiliten la construcción de un aprendizaje pertinente y actualizado de parte del alumnado. El Aprendizaje Basado en Problemas (ABP), siguiendo a Gómez, Ortuño y Miralles (2018), es una las estrategias didácticas más eficaces para desarrollar un aprendizaje histórico significativo a través de un trabajo colaborativo liderado por un grupo reducido de estudiantes (entre 6 y 9). Su dinámica consiste básicamente en abordar, a través de una rigurosa investigación, un problema relacionado con el futuro desempeño profesional de los y las estudiantes para buscar una posible solución a este (Núñez, Ávila, \& Olivares, 2017; Hung \& Amida, 2020; Ginaya, Kanca, \& Sri, 2020).

Dentro de la formación del profesorado de historia, el ABP se debe centrar necesariamente en el nuevo paradigma didáctico de la especialidad, el cual, según Seixas y Morton (2012), ya no se encuentra en el dominio de fechas, acontecimientos y personajes, sino que se focaliza en el desarrollo del pensamiento histórico, el cual posibilita la comprensión del pasado a partir de la interpretación de fuentes y de la creación de narrativas históricas.

Uno de los aspectos más significativos que debe considerar el docente al momento de implementar un ABP en la clase de historia es que no solo debe promover conocimientos de primer orden (el qué, dónde, cuándo o quién ha llevado a cabo las distintas acciones bajo un relato secuencial), sino que también debe privilegiar procedimientos y aprendizajes de segundo orden tales como el análisis de fuentes, la relevancia histórica, la dimensión ética y el tiempo histórico (Miralles \& Monteagudo, 2019; McClure \& Marino, 2020; Thorp \& Persson, 2020).

Para fomentar estas competencias históricas en el alumnado, es imprescindible que el profesor sea capaz de convertir una parte de las actividades comprendidas en el ABP en un proceso de aprendizaje por descubrimiento a través de un «laboratorio histórico». Este concepto es entendido como un lugar donde los estudiantes simulan el trabajo del historiador (Prats, 2011; Salazar, Orellana, Muñoz, \& Bellati, 2017; Álvarez, 2020) para analizar, bajo la guía del docente, diversas fuentes de información y elaborar los productos asociados al ABP, tales como el reporte de investigación y las posibles propuestas de mejora del problema estudiado.

A pesar del indiscutible aporte que puede tener el ABP en la formación de profesores de historia, no existen referentes empíricos en la literatura que puedan probar su eficacia en esta área; no obstante, hay varios trabajos teóricos (Gómez et al., 2017; Gómez et al., 2018) donde se señala que el $A B P$ se puede concebir dentro de la enseñanza de la historia como una metodología de indagación que permite cultivar habilidades sociales y desarrollar el pensamiento histórico en los alumnos y alumnas. Frente a este contexto, se hace necesario implementar y evaluar un ABP en la formación inicial de docentes de historia, razón por la cual se optó por implementar esta metodología en cinco asignaturas de dicha área para analizar su eficacia como estrategia didácticaevaluativa. 


\section{MATERIALES Y MÉTODOS}

Es un estudio exploratorio basado en una investigación-acción participativa que tiene como objetivo evaluar la experiencia de ABP aplicada entre marzo y diciembre de 2019 en una muestra de 144 estudiantes de Pedagogía de una universidad chilena. Esta muestra fue organizada en 16 grupos de 9 integrantes y tuvo un carácter intencional, pues este trabajo requirió de cinco asignaturas donde se desarrollen aprendizajes significativos relacionados con la historia chilena y mundial, y su respectiva enseñabilidad en el aula. El temario de trabajo consignado en el ABP fue el siguiente:

\section{CUADRO 1}

Ejes temáticos trabajados en el ABP por cada asignatura

\begin{tabular}{|c|c|}
\hline Asignatura & Temática \\
\hline $\begin{array}{l}\text { Cátedra I } \\
\text { Cátedra II }\end{array}$ & $\begin{array}{l}\text { Controversias históricas de la Conquista y Colonia de Chile (1540-1810), como, por ejemplo: la } \\
\text { prevalencia de la doctrina del descubrimiento de Chile en el curriculum; la violencia en el } \\
\text { proceso de mestizaje; la idealización de Pedro de Valdivia y Cristóbal Colón en las ilustraciones } \\
\text { de los textos escolares; y la omisión del pueblo mapuche como bastión de resistencia de la } \\
\text { conquista española. }\end{array}$ \\
\hline $\begin{array}{l}\text { Cátedra III } \\
\text { Cátedra IV }\end{array}$ & $\begin{array}{l}\text { Controversias históricas de la Independencia de Chile (1810-1823), como, por ejemplo: la } \\
\text { función patriótica de la historia escolar; la idealización de Bernardo O'Higgins y José Miguel } \\
\text { Carrera como los próceres de la patria; y la omisión de la mujer en los manuales escolares } \\
\text { durante el proceso independentista. }\end{array}$ \\
\hline Cátedra V & $\begin{array}{l}\text { Controversias históricas de las grandes civilizaciones de la Antigüedad, como, por ejemplo: la } \\
\text { representación discursiva de la mujer de la Grecia Antigua en los textos escolares chilenos; el } \\
\text { predominio del adultocentrismo en el curriculum escolar; el rol del infante en el Imperio } \\
\text { Romano; el eurocentrismo; y la omisión de la China Antigua en las bases curriculares de } \\
\text { Enseñanza Básica. }\end{array}$ \\
\hline
\end{tabular}

Diagnóstico del problema: Para este apartado, se utilizó un KPSI que tuvo como propósito averiguar los conocimientos previos de los futuros docentes sobre las temáticas previstas para cada curso de la muestra y los recuerdos que tienen acerca de la metodología utilizada por sus profesores de Enseñanza Básica, Media y Superior para enseñar estos contenidos. Esta evaluación inicial fue útil para diagnosticar que el $90,2 \%$ de los estudiantes recuerdan que sus profesores le enseñaron la historia de Chile y de la Antigüedad clásica mediante clases tradicionales, centradas fundamentalmente en la lección expositiva y en el trabajo individual; asimismo, permitió visualizar que todos los cursos coincidieron en que la enseñanza de los contenidos previstos se han impartido desde un paradigma cognitivo centrado en la memorización de aprendizajes de primer orden relacionados con fechas, hitos y personajes específicos.

Diseño de la experiencia: Para tratar de solucionar en parte el problema diagnosticado, se diseña una propuesta de innovación basada en la implementación de un ABP. Para tal propósito, el alumnado se rigió por el siguiente itinerario de trabajo:

Fase I. Preparación: En las tres semanas previas a la actividad, el profesor explica todos los contenidos relativos a la metodología de trabajo del ABP y a los principales lineamientos de las problemáticas trabajadas. Luego, se conforman los equipos de trabajo por afinidad y se asignan los siguientes roles para cada integrante: portavoz, secretario, investigador principal, investigador alterno y colaborador(es). 
Fase II. Selección de la problemática: Los grupos eligen un tema de interés social y que se enmarque dentro de los ejes temáticos consignados para cada curso. Para ello, se recomienda tener como referencia alguna experiencia de sus prácticas pedagógicas progresivas o revisar el curriculum escolar para delimitar una controversia histórica que consideren necesaria replantear desde enfoques historiográficos innovadores (perspectiva de género, visiones extra-europeas, cosmovisión indígena, relaciones de alteridad, el presente en clave histórica, entre otras).

Fase III. Laboratorio de Historia: Los estudiantes desarrollan un laboratorio histórico (Salazar et al., 2017), que les permite simular el trabajo del historiador en el aula a partir de la búsqueda, selección y tratamiento de fuentes. Dentro de este laboratorio, los alumnos deberán aplicar las siguientes cuatro heurísticas:

- Heurística de origen, teniendo en cuenta de donde proviene el documento histórico y su propósito.

- Contextualización, con el fin de colocar el documento en su contexto temporal y espacial.

- Lectura cerrada, para extraer la principal idea del documento.

- Corroboración heurística, para comparar las múltiples fuentes.

Fase IV. Elaboración del informe: Los alumnos elaboran un reporte de investigación sobre el objeto de estudio. Este debe tener la siguiente estructura: portada, introducción, preguntas de investigación, objetivos, metodología, desarrollo de la temática, conclusiones, bibliografía y anexos.

Fase V: Diseño de la propuesta didáctica: El estudiantado elabora una propuesta didáctica que permita enseñar la problemática seleccionada desde un enfoque innovador y constructivista. Se debe regir por el formato entregado por el docente.

Fase VI: Simulación de clase: A partir de la propuesta didáctica elaborada, uno de los integrantes de cada equipo debe realizar una simulación de clase con los principales resultados de la investigación. La simulación debe contar con material de apoyo, respetar la estructura de la clase y no superar los 30 minutos de duración.

Fase VII. Entrega del registro de campo: Cada estudiante deberá entregar un registro de campo donde conste su percepción sobre la experiencia.

Posteriormente, se procedió a la validación del instructivo del ABP por dos expertos universitarios en Didáctica de la Historia. Las recomendaciones dadas por estos especialistas fueron fundamentales para mejorar el protocolo de la actividad.

Implementación del ABP: La actividad se llevó a cabo en el horario habitual de las asignaturas previstas (dos veces a la semana), y se realizó durante 35 clases de 120 minutos cada una ( 7 clases por curso), correspondientes a 70 horas de intervención en el aula. La implementación del ABP se fundamentó en las fases operativas mencionadas en el diseño de la actividad.

Evaluación de la experiencia: El análisis evaluativo se basa en los datos provistos por una encuesta tipo Likert elaborada para conocer la percepción del alumnado sobre la propuesta. Este instrumento cuenta con 10 reactivos (cuadro 2 ) y cinco opciones de respuesta: $1=$ Totalmente en desacuerdo; $2=$ En desacuerdo; 3=Ni de acuerdo, ni en desacuerdo; 4= De acuerdo; $y$ 5= Totalmente de acuerdo. 


\section{CUADRO 2}

Reactivos de la encuesta tipo Likert

\begin{tabular}{l}
\hline 1. Las clases que contemplaron la preparación del ABP favorecieron la comprensión de los contenidos. \\
\hline $\begin{array}{l}\text { 2. El laboratorio histórico implementado en el aula fue una instancia predilecta para investigar sobre el objeto de } \\
\text { estudio. }\end{array}$ \\
\hline 3. El trabajo grupal realizado fue efectivo para potenciar el pensamiento histórico y mejorar las habilidades sociales. \\
\hline 4. La estrategia fue útil para aprender contenidos actualizados sobre las temáticas históricas analizadas. \\
\hline 5. El ABP permitió reflexionar sobre la problemática y proponer propuestas o lineamientos de mejora. \\
\hline 6. El ABP fomenta el trabajo autónomo, motiva la participación en clases y mejora el clima de aula. \\
\hline 7. La metodología utilizada favorece el acercamiento entre la teoría y la práctica. \\
\hline 8. El proceso evaluativo realizado por el docente fue una pieza clave para mejorar constantemente el trabajo. \\
\hline 9. El ABP es una estrategia pertinente, eficaz e innovadora para utilizar con sus futuros estudiantes. \\
\hline 10. Te gustaría seguir desarrollando este tipo de experiencia durante tu formación universitaria. \\
\hline
\end{tabular}

Para enriquecer el análisis de los datos arrojados por esta encuesta de satisfacción, se desarrolla un proceso de triangulación de múltiples fuentes que complementa y valida dichos antecedentes con un análisis documental que permitió evaluar la información contenida en los demás insumos utilizados, es decir, los cuadernos de campo de los estudiantes, el registro de asistencia, la rúbrica diseñada para evaluar el ABP y el diario del investigador que fue elaborado para analizar la experiencia.

\section{RESULTADOS}

A partir de la perspectiva general del alumnado, los resultados obtenidos en la experiencia fueron positivos, ya que el $100 \%$ de las valoraciones de los estudiantes para el conjunto de los indicadores consultados estuvieron en el rango: ni de acuerdo, ni en desacuerdo, de acuerdo o totalmente de acuerdo (figura 1).

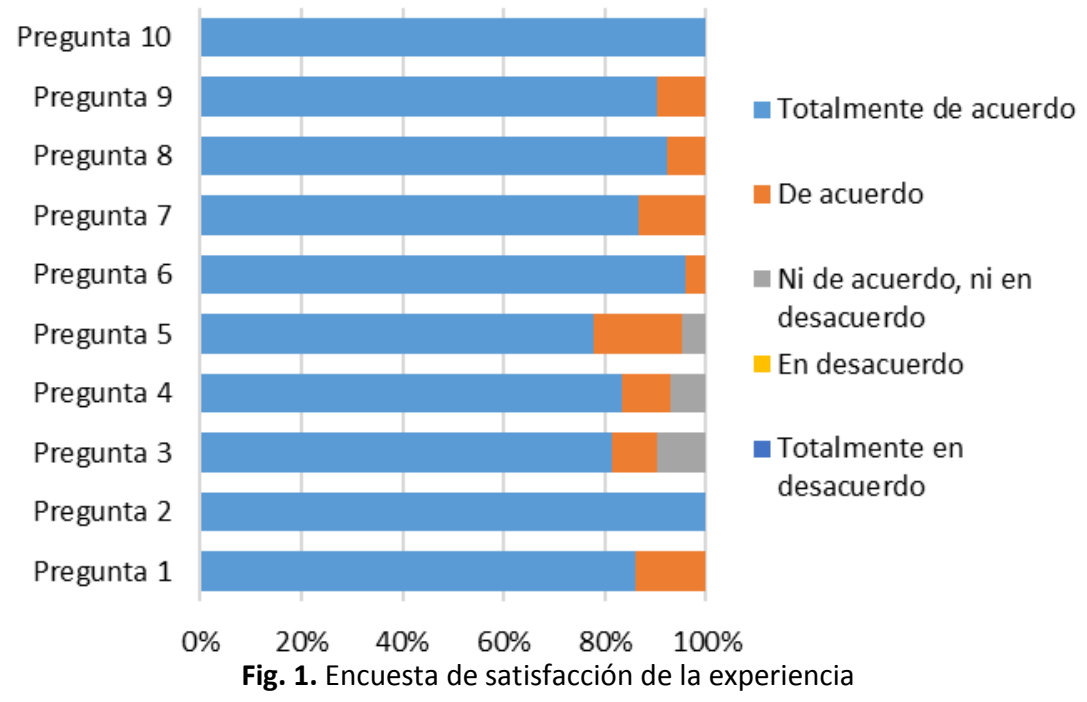

Las puntuaciones más altas son para los reactivos $2,6,8$ y 10, los cuales aluden a la importancia del laboratorio histórico implementado, a los beneficios de utilizar el ABP en el aula, al proceso evaluativo realizado por el profesor y a las expectativas de utilizar dicha estrategia en otras asignaturas. 
Desde la perspectiva docente, un primer rasgo interesante a destacar del ABP es la escasa cercanía que tiene el alumnado con dicha estrategia. Esto se refleja, fundamentalmente, en las dificultades que tuvieron 8 equipos para asimilar la metodología de trabajo, puesto que algunos de sus integrantes no conocían la estructura del $A B P$, o nunca habían participado en uno.

Otras de las dificultades constatadas se relacionan con la simulación de clase, última instancia del $A B P$, donde los sujetos participantes tuvieron que presentar el problema investigado y proponer una posible solución al mismo. Entre los principales inconvenientes enunciados se encuentra el olvido de determinados sustentos históricos de la problemática, el escaso bagaje erudito y la inseguridad del alumnado para responder las preguntas de los compañeros y del profesor.

Asimismo, cabe destacar que otro de los problemas evidenciados se vincula con la sobrecarga de trabajo que implica el ABP, pues, en el caso de los cursos consignados, exigió la realización de todas las fases operativas especificadas en el diseño de la experiencia.

Otra dificultad observada es la falta de cohesión en el trabajo grupal. Al respecto, un estudiante señala que "al ser un trabajo en grupo, siempre hay personas que trabajan más que otras, por lo que es un poco agotador no tener el apoyo de todos los integrantes" (P98). Otro estudiante comparte esta postura y sostiene que "la principal dificultad que tuvimos fue la inasistencia de algunos compañeros, ya que, en dos ocasiones, el grupo no se encontró en su totalidad para continuar de forma exitosa con la preparación del ABP" (P12).

A pesar de las complicaciones mencionadas, el 83,3\% de los estudiantes, según la encuesta aplicada, está totalmente de acuerdo en que la experiencia fue útil para aprender contenidos actualizados sobre las problemáticas analizadas, ya que les permitió desarrollar aprendizajes significativos a través del laboratorio histórico realizado. Gracias a esta instancia, pudieron elaborar explicaciones complejas en torno a los cimientos de sus temáticas y fueron capaces de dimensionar la relevancia histórica de los problemas investigados.

Por este motivo, y tal como indica la encuesta, el $100 \%$ de los alumnos están totalmente de acuerdo en que el ABP se debe implementar de forma más frecuente dentro de la universidad, pues, según su percepción general, es importante crear espacios que contribuyan a potenciar competencias que promuevan el pensamiento histórico, especialmente cuando se constata que la enseñanza de la historia en el sistema educativo se centra, fundamentalmente, en un enfoque nacionalista, adultocentrista y androcéntrico.

Esta estrategia se debiera trabajar de manera más recurrente en la formación docente, ya que entre más metodologías aprendamos a manejar, mayor será la calidad de nuestra intervención en la enseñanza de la disciplina (P122).

La investigación y la posterior simulación de clase que llevamos a cabo sobre el rol del infante y/o adolescente en las civilizaciones antiguas nos permitió evidenciar que la asignatura de historia se ha enseñado desde una perspectiva adultocéntrica, obviando u omitiendo que grandes personajes lograron hazañas significativas cuando todavía eran niños o adolescentes, tal como ocurrió con Tutankamon (Egipto), Augusto Rómulo (Roma), Alejandro Magno (Grecia) y Ying Zheng (China) (P51).

La indagación que realizamos a través del ABP sobre las representaciones discursivas de la mujer de la Antigua Grecia en un texto escolar de séptimo básico nos llevó a concluir que la enseñanza de la historia se concibe desde 
un enfoque unilateral donde la perspectiva masculina se presenta como la medida de todas las cosas, lo cual puede generar conductas androcéntricas o la asimilación de estereotipos sexistas en los estudiantes desde edades muy tempranas (P76).

Por otra parte, también es importante mencionar que el ABP fue muy eficaz para potenciar los valores y cultivar las habilidades blandas de los estudiantes, como la proactividad, la responsabilidad, el trabajo colaborativo y la ética profesional. De hecho, los estudiantes reconocen que esta estrategia fomenta varias cualidades como la motivación y el trabajo autónomo.

Como primer aspecto positivo puedo destacar la instancia que ofreció el profesor para investigar problemáticas de interés personal, lo que causa una mayor motivación a la hora de realizar el trabajo (P3).

La autonomía que nos entrega el docente a la hora de seleccionar la problemática, a partir del análisis de los distintos insumos curriculares, me permitió percatarme de algunas temáticas innovadoras y poco estudiadas que se podrían considerar en la enseñabilidad de la historia, tales como el adultocentrismo en la historia escolar y las representaciones de género en los libros de texto (P52).

Asimismo, destaca el elevado nivel de compromiso y responsabilidad con el que los estudiantes realizaron sus respectivos trabajos; ello se observa en que el 95,8\% cumplió con el desarrollo de la actividad. El compromiso con la experiencia también se manifestó en que el 84,7\% del alumnado asistió de forma regular a las clases que contemplaron la preparación y exposición del ABP.

En cuanto al desempeño académico logrado, destaca el 86,8\% de aprobación por parte de los sujetos participantes, evidenciándose una mejora importante en los resultados obtenidos en una prueba escrita tradicional con contenidos similares, donde el porcentaje de aprobación eran cercanos al 75\% para las asignaturas en las cuales se aplicó esta experiencia. En este caso concreto, se obtuvieron resultados positivos que se ven reflejados en que el $86,8 \%$ de las calificaciones superan el 4,0 en una escala de 1,0 a 7,0.

Igualmente, resulta conveniente precisar que la actividad ha reforzado la estrecha relación entre el docente y los alumnos, porque se convirtió en un espacio de mayor interacción entre las partes. De hecho, uno de los aspectos más valorados por los estudiantes es el proceso de evaluación formativa realizado a lo largo del trabajo y la iniciativa del profesor de disponer de varias clases para preparar el ABP. Al respecto, un estudiante señala que "durante toda la actividad se fueron realizando revisiones por parte del docente, lo que generó que se presentaran varias oportunidades para ir mejorando el trabajo antes de que fuera calificado" (P3). Otro estudiante, respecto al segundo punto, plantea que "el hecho de que se dispusieran de varias clases para preparar el ABP es un aspecto muy positivo porque permitió contrarrestar la sobrecarga de trabajo" (P144).

También es importante resaltar que el 90,2\% del profesorado en formación está totalmente de acuerdo en utilizar el ABP con sus futuros estudiantes, pues la mayoría no ha tenido la oportunidad de trabajar en uno durante su formación universitaria o de implementarlo en sus prácticas progresivas. 
El aprendizaje que se puede adquirir por medio de esta estrategia es significativo y altamente pertinente, ya que contribuye a cambiar el modelo de enseñanza tradicional y a potenciar el trabajo autónomo. Asimismo, creo que esta estrategia es muy útil a la hora de implementar una clase como futura docente porque fomenta el desarrollo de los aprendizajes de segundo orden del pensamiento histórico (P70).

No obstante, a pesar de las amplias ventajas de aplicar el ABP con los futuros estudiantes, el profesorado en formación considera que existen diversas condicionantes que se deben considerar con respecto al contexto de aula, tales como el elevado número promedio de estudiantes por grupocurso y la madurez cognitiva del alumnado.

Entre los principales retos que debe enfrentar el profesor en el aula al momento de implementar el $A B P$, se encuentran el gran número de estudiantes por curso (entre 35 y 45 alumnos) y los posibles problemas de comportamiento que se pudieran generar en el aula, ya que dicha metodología puede provocar distracción, aburrimiento y estrés en el alumnado debido a que requiere la ejecución de diferentes labores (P12).

A modo personal, creo que esta estrategia puede servir para realizar un excelente trabajo en el aula, pero con niños y niñas que pertenezcan al segundo ciclo de Enseñanza Básica debido al nivel de complejidad de las diversas tareas involucradas (P111).

\section{DISCUSIÓN}

Si bien la experiencia generó resultados positivos en cuanto al desarrollo de habilidades sociales y de aprendizajes históricos y pedagógicos en el alumnado, es importante atenuar las limitaciones observadas en el ABP implementado y mejorar su potencial didáctico-evaluativo en futuras intervenciones. Por esta razón, desde la perspectiva docente, es necesario cautelar los siguientes aspectos: el gran volumen de trabajo que implica para el docente y el estudiante; el poco interés de algunos alumnos por la temática y/o la estrategia; el escaso conocimiento que existe sobre la mayoría de las temáticas abordadas; y el dificultoso acceso a fuentes primarias para investigar las problemáticas.

Uno de los aspectos enunciados que más visualizan otros estudios sobre experiencias implementadas en diferentes carreras es el elevado volumen de trabajo que significa para el profesorado universitario. Para Latasa, Lozano y Ocerinjauregi (2012) y Ginaya et al. (2020), el ABP supone una tarea muy costosa en tiempo y extremadamente compleja, por lo que resulta imprescindible abordar la creación de equipos docentes, y su respectiva coordinación, para aunar esfuerzos en torno al diseño, implementación y evaluación de propuestas basadas en dicha metodología.

A pesar de las dificultades consignadas, los resultados observados en el área de desarrollo de habilidades blandas son positivos y coherentes con los obtenidos en otras experiencias de ABP realizadas en carreras universitarias de Chile, Ecuador e Indonesia como Educación Diferencial (Jofré \& Contreras, 2013), Psicología Clínica (Palta, Sigüenza, \& Guevara, 2018), Kinesiología (Sepúlveda, Cabezas, García, \& Fonseca, 2019) y Turismo Industrial (Ginaya et al., 2020), ya que, tal como se señaló para este caso particular, la estrategia logró aumentar el compromiso del alumnado con su propio aprendizaje y promover el trabajo colaborativo. 
Sin embargo, no es posible contrastar los resultados de la experiencia con otros estudios en cuanto al logro de aprendizajes históricos y didácticos específicos de esta disciplina, puesto que no existen referentes empíricos en la literatura especializada que permitan evaluar la eficacia del ABP en esta área. Por tal motivo, esta experiencia de innovación docente constituye un primer acercamiento para mejorar la formación de docentes de historia a través de la implementación de la metodología prevista.

Bajo dicha premisa, y si bien la presente investigación evidenció la eficacia del ABP para potenciar habilidades sociales y mejorar la comprensión sobre la historia y su didáctica, es importante continuar desarrollando nuevos estudios para profundizar en esta temática, o bien para analizar experiencias similares que tengan la pretensión de innovar en la enseñanza de la especialidad.

Por último, cabe destacar que la presente propuesta ofrece lineamientos metodológicos que pueden ser adaptados y replicados para crear experiencias de ABP que se pueden implementar en diversos contextos educativos.

\section{AGRADECIMIENTOS}

Esta investigación se enmarca en el Proyecto NI-EDU 02/2020 "El uso del Aprendizaje Basado en Problemas (ABP) en la enseñanza de la historia. Potencialidades, problemas y desafíos" adscrito al PI FID USC 1897 de la Facultad de Educación de la Universidad Católica de la Santísima Concepción, Chile. Se agradece a la institución patrocinante por el apoyo otorgado.

\section{ÉTICA, CONFLICTO DE INTERESES Y DECLARACIÓN DE FINANCIAMIENTO}

El autor declara haber cumplido con todos los requisitos éticos y legales pertinentes, tanto durante el estudio como en el manuscrito; que no hay conflictos de interés de ningún tipo, y que todas las fuentes financieras se detallan plena y claramente en la sección de agradecimientos. Asimismo, están de acuerdo con la versión editada final del documento. El respectivo documento legal firmado se encuentra en los archivos de la revista.

El porcentaje de contribución total para la conceptualización, preparación y corrección de este artículo fue H.A.A.S $100 \%$.

\section{REFERENCIAS}

Álvarez, H. (2020). El uso del debate en la Educación en Derechos Humanos. Problemas, desafíos y potencialidades. Mendive, 18(2), 219-234.

Barca, I. (2011). Narrativas e consciência histórica dos jovens. Enseñanza de las Ciencias Sociales, (10), 23-29.

Barton, K. (2010). Investigación sobre las ideas de los estudiantes acerca de la historia. Enseñanza de las Ciencias Sociales, (9), 97-114.

Ginaya, G., Kanca, N., \& Sri, N. (2020). Designing problem-based learning (PBL) model for tourism vocational education in 4.0 industry. International Journal of Linguistics, Literature and Culture, 6(1), 14-23. DOI: 10.21744/ijllc.v6n1.808

Gómez, C., López, R., Miralles, P., \& Prats, J. (2017). Enseñanza de la historia y competencias educativas. Barcelona: Graó.

Gómez, C., Ortuño, J., \& Miralles, P. (2018). Enseñar ciencias sociales con métodos activos de aprendizaje. Reflexiones y propuestas a través de la indagación. Barcelona: Octaedro. 
González, G., Santisteban, A., \& Pagès, J. (2020). Finalidades de la enseñanza de la historia en futuros profesores. Magis, Revista Internacional de Investigación en Educación, 13, 1-23. DOI: 10.11144/Javeriana.m13.fehf

Hung, W., \& Amida, A. (2020). Problem-Based Learning in college science. In J. Mintzes \& E. Walter (Eds.), Active learning in college science (pp. 325-340). Cham, Switzerland: Springer.

Jofré, C., \& Contreras, F. (2013). Implementación de la metodología ABP (Aprendizaje Basado en Problemas) en estudiantes de primer año de la carrera de Educación Diferencial. Estudios Pedagógicos, 39(1), 99-113. DOI: 10.4067/S0718-07052013000100006

Latasa, I., Lozano, P., \& Ocerinjauregi, N. (2012). Aprendizaje Basado en Problemas en currículos tradicionales: beneficios e inconvenientes. Formación universitaria, 5(5), 15-26. DOI: 10.4067/\$0718-50062012000500003

McClure, D., \& Marino, M. (2020). Historical thinking and sports history: a case study using the Brooklyn Dodgers. The Social Studies, 111(3), 109-122. DOI: 10.1080/00377996.2019.1692186

Miralles, P., \& Monteagudo, J. (2019). Métodos, instrumentos y procedimientos para conocer cómo se evalúan las competencias históricas. Educar em Revista, 35(74), 127-144. DOI: 10.1590/0104-4060.64404

Núñez, S., Ávila, J., \& Olivares, S. (2017). El desarrollo del pensamiento crítico en estudiantes universitarios por medio del Aprendizaje Basado en Problemas. Revista Iberoamericana de Educación Superior, 8(23), 84-103.

Palta, N., Sigüenza, J., \& Guevara, C. (2018). Aprendizaje basado en problemas en la carrera de Psicología Clínica. Revista Electrónica de Psicología Iztacala, 21(4), 1411-1433.

Prats, J. (2011). Didáctica de la Geografía y la Historia. Barcelona: Graó.

Prats, J. (2017). Retos y dificultades para la enseñanza de la historia. En P. Sanz, J. Molero \& D. Rodríguez (Eds.), La historia en el aula. Innovación docente y enseñanza de la historia en la educación secundaria (pp. 15-32). Lleida: Milenio.

Sáez, A., Parra-Díaz, J., \& Herrera, F. (2020). Autocognición sobre civismo y ciudadanía del estudiantado de Pedagogía en Historia, Geografía y Educación Cívica en una universidad chilena. Revista Actualidades Investigativas en Educación, 20(1), 1-24. DOI:10.15517/AIE.V20I1.40144

Salazar, R., Orellana, C., Muñoz, C., \& Bellati, I. (2017). El aula como laboratorio histórico: la Guerra Civil Española. En C. Gómez, R. López, P. Miralles \& J. Prats (Eds.), Enseñanza de la historia y competencias educativas (pp. 110-126). Barcelona: Graó.

Seixas, P., \& Morton, T. (2012). The big six historical thinking concepts. Toronto: Nelson.

Sepúlveda, P., Cabezas, M., García, J., \& Fonseca, F. (2019). Aprendizaje basado en problemas: percepción del proceso enseñanza aprendizaje de las ciencias preclínicas por estudiantes de Kinesiología. Educación Médica. DOI: 10.1016/j.edumed.2019.01.004

Thorp, R., \& Persson, A. (2020). On historical thinking and the history educational challenge. Educational Philosophy and Theory, 52(8), 891-901. DOI: 10.1080/00131857.2020.1712550 